\title{
A Proposed Mesh Placement for Repair of Ventral Hernia and Contouring of the Abdomen
}

\author{
Adel Tolba', Wael Shelfa' ${ }^{2}$, Hazem Nour' ${ }^{2}$, Hessen Yaser ${ }^{2}$, Mansour Salah $^{2}$ \\ ${ }^{1}$ Plastic Surgery Unit, Zagazig University, Zagazig, Egypt \\ ${ }^{2}$ Gastrointestinal Surgical Unit, Zagazig University, Zagazig, Egypt \\ Email: dadlahm@yahoo.com
}

Received 9 March 2015; accepted 19 October 2015; published 22 October 2015

Copyright (C) 2015 by authors and Scientific Research Publishing Inc.

This work is licensed under the Creative Commons Attribution International License (CC BY). http://creativecommons.org/licenses/by/4.0/

c) (i) Open Access

\begin{abstract}
Introduction: Abdominal wall defects and muscle redundancy in the form of protruded abdomen still represent a challenging problem. The aim of this article is to reconstruct the abdominal wall and regain the abdominal contour through the application of biosynthetic meshes in certain fashion. Patients and Methods: this is a prospective study done 25 patients with long standing ventral hernia and lax abdominal muscle wall. All patients were performed at Zagazig university hospitals in the period between September 2011 and November 2013. Polypropylene mesh is used for every patient after anatomical hernia repair for hernioplasty and then shaping like outstretched hand (fingers processes). The aim is to reinforce the abdominal wall and reshape the abdomen. Results: regarding to the aesthetic aspect and success rate of the abdominal wall reconstruction, we have a good satisfactory results without recurrence in a period of 2 years follow up. Three patients are suffered from wound infection and partial edge ischemia in two patients. Bleeding and delayed wound healing in ten patients and persistent seroma are occurred in fife patients. Conclusion: It is safe with a good patient satisfaction to combine ventral hernia repair and abdominoplasty in cases with excess skin and musculofascial laxity of the abdominal wall (pendulous abdomen) with application of this finger processes mesh, after following the proper technique and precautions.
\end{abstract}

\section{Keywords}

Abdominoplasty, Pendulous Abdomen, Contour

\section{Introduction}

The term abdominoplasty covers a range of surgical procedures including dermolipectomy, liposuction and

How to cite this paper: Tolba, A., Shelfa, W., Nour, H., Yaser, H. and Salah, M. (2015) A Proposed Mesh Placement for Repair of Ventral Hernia and Contouring of the Abdomen. Surgical Science, 6, 446-453.

http://dx.doi.org/10.4236/ss.2015.610064 
dealing with musculofascial abdominal wall layer [1]. Fowler at 1994 had stated that the abdominal contouring was shaped through a proper relationship between the osteomuscular system, the subcutaneous adipose tissue and the quantity of skin.

Decisions regarding technique are based on an assessment of the overall clinical status of the patient, the location and size of the defect and well as extension of layers involved, and etiology. The incidence of ventral hernias as a complication of abdominal operations has been reported as high as $20 \%$ [2].

Many indications for ventral hernia repair like the generalized pain and fair of strangulations also the contouring of the abdomen are considered the most common indications for repair of ventral hernia. Open or laparoscopic prosthetic mesh placement is the surgical approaches currently used. The choice of one approach over the other varies on a case-by-case basis as well as surgeon preference. Open techniques may range from simple herniorraphy, fascial plication, components separation, and mesh placement as an underlay, retro-rectus or overlay [3].

In this thesis, we tried to evaluate patients with pendulous abdomen or complex abdominal wall defects and add a novel procedure for application of the mesh as a method for abdominal wall reconstruction and re-contouring the abdomen.

\section{Patients and Methods}

This thesis was a prospective randomized study done on 25 patients in the period between 2011 and 2013, patients ages ranged from 35 and 45 years, female to male ratio was $4 / 1$ (20 female and 5 male) in Table 1 . All patients complained of abdominal contour deformity (pendulous abdomen) either alone in 10 patients or in association with abdominal hernias in 15 patients. The follow up period ranged from 12 and 28 months.

Exclusion criteria patients with systemic diseases like ischaemic heart diseases and obstructive lung disease, also patients with flappy abdominal wall due to systemic diseases like Myasthenia gravis. Patients with incisional hernia and previous transverse scars were also excluded.

Full history taking, pre-operative preparation, photography and measuring the abdominal girth are done for every patient (Table 2).

Table 1. Preoperative patients characters showing the age sex and the associated risk factors.

\begin{tabular}{cc}
\hline & Patents Characters \\
\hline Age; & $\begin{array}{c}\text { Range }(33-45) ; \text { Mean } 33+2.1 \\
\text { M/F ratio; } 1 / 4(5-20)\end{array}$ \\
Pendulous abdomen with ventral hernia & $\mathrm{n}=15$ \\
Without ventral hernia & $\mathrm{n}=10$ \\
Hernia defects & Range (3 to 10$) \mathrm{cm}^{2}$ \\
Associated risk factors & Diabetes $\mathrm{n}=7$ \\
& Smoking $\mathrm{n}=2$ \\
& chest asthma $\mathrm{n}=4$ \\
\hline
\end{tabular}

Table 2. Postoperative patients characters.

\begin{tabular}{cc}
\hline Postoperative Complication & No. (\%) \\
Wound infection & $3(12 \%)$ \\
Seroma & $5(20 \%)$ \\
Central flap Ischemia $(2 \times 3 \mathrm{~cm})$ & $3(12 \%)$ \\
Full thickness necrosis & $2(8 \%)$ \\
Anesthesia of the central infra Umbilical regions & $2(2 \%)$ \\
Bleeding & $5(20 \%)$ \\
Persistent pain and discomfort & $2(8 \%)$ \\
Recurrence & 0
\end{tabular}




\section{Technique}

A lower abdominal crease incision as the procedure of dermo-lipectomy and complete flap elevation till the xyphoid process with minimal dissection of the lateral supra-umbilical region.

Undermining is done in an inverted V-type fashion. The undermining in this fashion keeping us away from the costal margin, preserving the blood supply coming to the upper flap from the lateral side (segmental perforators) (Figure 1 and Figure 2).

The hernia defect is repaired anatomically, then the mesh is introduced after its cutting in a fingers like processes, the stem of the mesh in applied at the most lateral aspect beyond the rectus sheath and the three processes were directed medially with one upward, one transverse and the third downward to be continued with that at the other side in an interrupted sutures above, at and below the umbilicus (Figure 3 and Figure 4). To achieve a narrow waist, more interrupted sutures were fixed most laterally and strengthen the rectus sheath with two longitudinal sheets of meshes (Figure 5 and Figure 6).

Light dressing is used. A compression garment is applied immediately following the operation. Early ambulation is important to avoid deep venous thrombosis. The suction drains are removed when the daily drainage is less than 50 cc. Sutures removed 10 days following the operation. Abdominal binder is worn for 3 months. Regular follow-up on an outpatient basis was done every week for one month to detect early complications (seroma, or infection) then monthly thereafter for 6 months. Post-operative photography is taken for comparison (Figure 7 and Figure 8). It is better to be at least 2 months after operation to give a chance for the oedema to resolve.

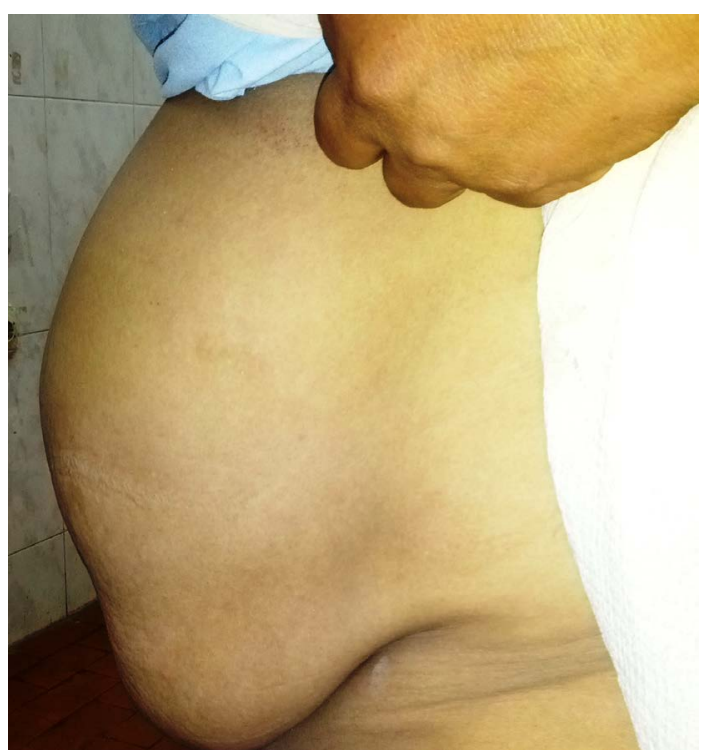

Figure 1. Pendulous abdomen.

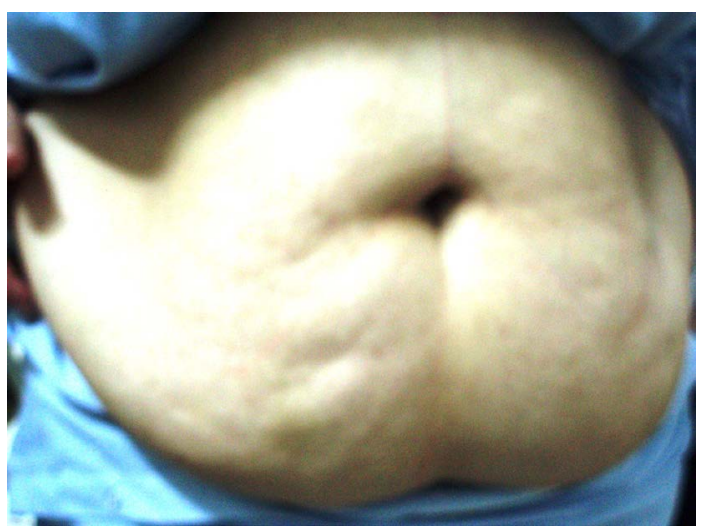

Figure 2. Redundant abdomen. 


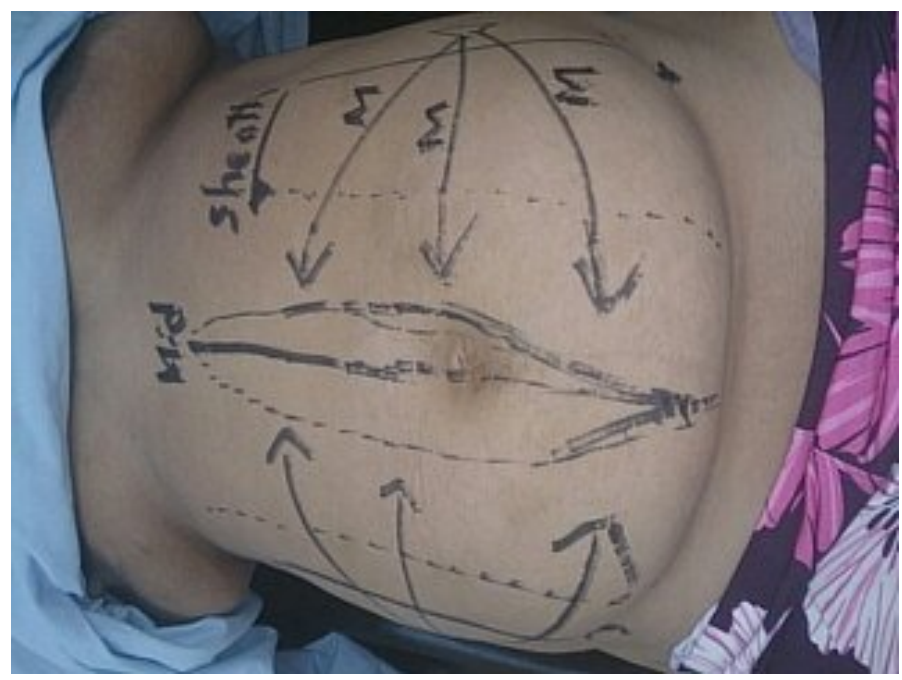

Figure 3. Marking the mesh placement.

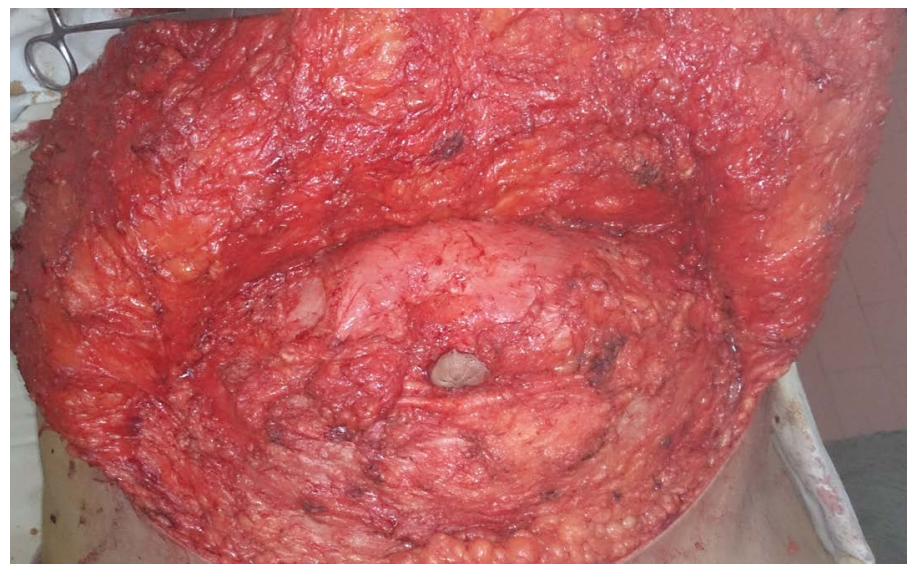

Figure 4. Picture after complete elevation of the abdominal wall away from costal margins.

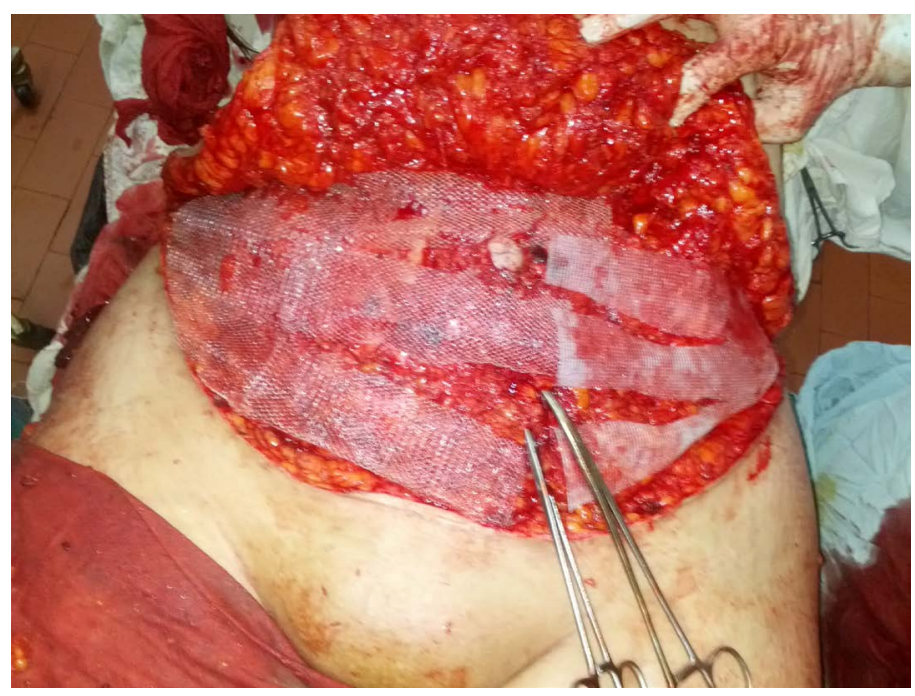

Figure 5. Mesh placement technique like fingers in both sides of the abdominal wall. 


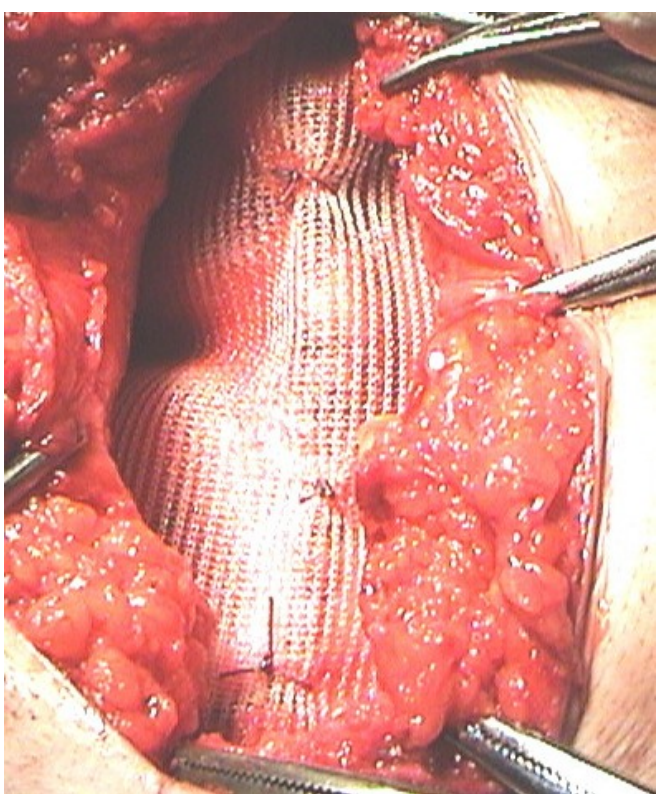

Figure 6. Longitudinal mesh placement at midline.

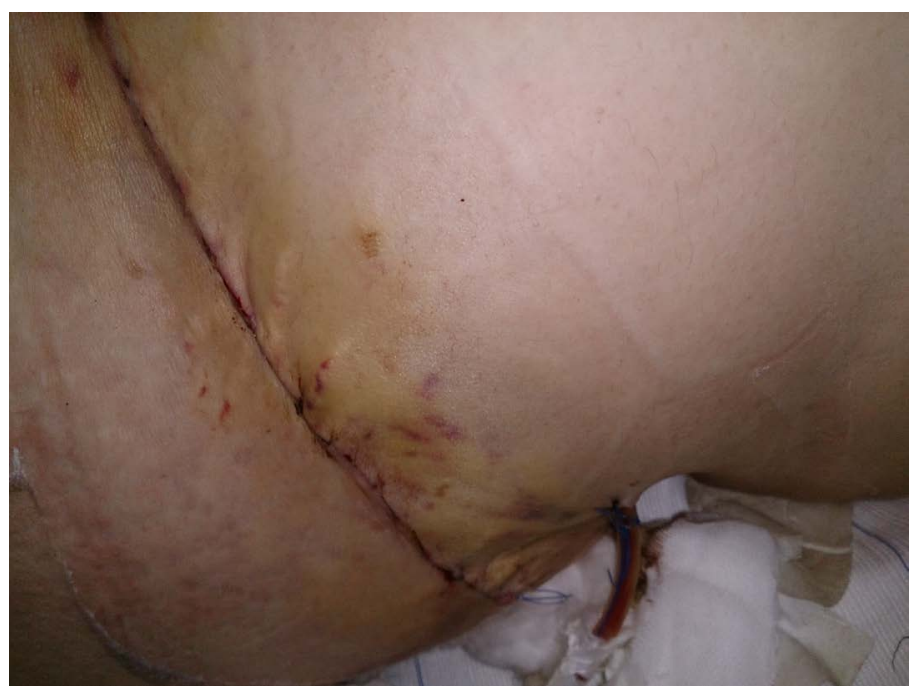

Figure 7. Immediate postoperative results with a waist forming.

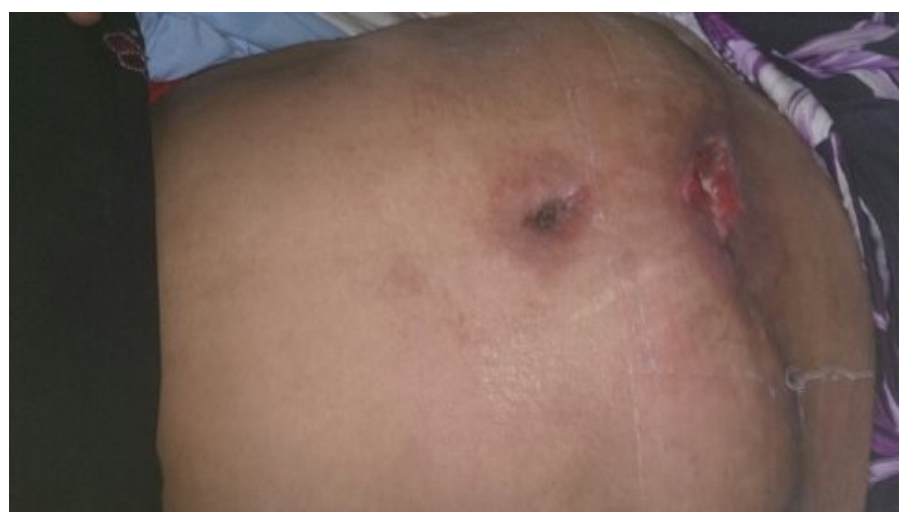

Figure 8. Central partial skin loss. 


\section{Results}

This study was done on 25 patients complaining of abdominal contour deformities in the form of bulging, redundancy, either alone or in conjunction with hernia. 20 patients were females and 5 males. Five females and two males were diabetics

Patient satisfaction was achieved in 20 patients 16 females and 4 males (80\%) in Table 3 . The complications met in the study were in the form of central flap loss in 2 patients $(2 \times 3 \mathrm{~cm})$, persistent anaesthesia of the central infra-umbilical region for 8 months in one patient. Persistent seroma in 5 patients for maximum three months. Minor wound infection occurred in 3 patients. The cases of central flap loss was managed by surgical debridement and secondary sutures. The patients with seroma were treated by frequent aspiration drainage and compression.

\section{Discussion}

The first dermo-lipectomies of the abdominal wall were performed by surgeons who were repairing massive umbilical hernias. Subsequently in the evolution of the technique of dermo-lipectomy of the abdominal wall, three methods have been advocated 1) vertical midline resection; 2) transverse resection; and 3) a combination of the vertical and transverse methods. During the period 1960's to the 1980's, it became obvious that low transverse incision was the preferred choice for patients undergoing abdominoplasty [4].

This study was done to assess the need and benefits of concomitant hernia repair with musclofascial mesh plication. After reviewing the results of the study it appeared that it is possible to do combined dermolipectomy and waist creating with mesh plication though the finger processes fashion which avoid the increased of intra-abdominal pressure and respiratory problem ,also it created graduated tension around the umbilicus for waist formation .

Rozen et al. [5] [6] reported that the two most commonly used prosthetic materials are polypropylene and expanded polytetrafluoroethylene (ePTFE). It is equivalent to polypropylene in terms of suture retention strength. As a result of its flexibility, conforming nature, and minimal tissue in-growth, ePTFE can be placed directly on bowel. The material is virtually impenetrable, preventing host tissue ingrowth and often leading to seroma formation. Synthetic mesh offers high tensile strength, however it is associated with chronic inflammatory response and scar formation that subsequently promotes visceral tissue ingrowth into mesh porosities that can lead to complications such as adhesions to the mesh, bowel obstruction, and enterocutaneous fistula.

There is no enough data to support the use of absorbable over non-absorbable prosthesis regard to the complication rate. We used the polypropylene mesh in all our patients without significant complications related to the mesh (0\%). This technique as described first exposes the anterior sheath and the aponeurosis of the external oblique muscle postoperative complications of the technique mostly seroma (2\%).

Ramirez et al. introduced the components separation technique (CST) that involved the release of the posterior rectus sheath in addition to the external oblique fascia [6] [7]. This method uses bilateral, innervated, bipedicle, muscle flaps, advanced medially to reconstruct the central abdominal wall defects. The components separation technique represents a landmark for the tissue transfer techniques used to repair complex abdominal midline defects.

In our study the fascial defects varies from 5 to $9 \mathrm{~cm}$ and all hernia repair using the prosthetic mesh agree with most surgeons who still believe prosthetic mesh repair is the standard of care for the non complex hernia patient with a fascial defect greater than $2 \mathrm{~cm}$. Also there are no enough number of randomized controlled trials that support the use of mesh versus primary repair for non complex hernias with defects sizing $6 \mathrm{~cm}$ or less [8] [9].

Table 3. Post operative patients satisfaction (three grades) excellent satisfaction $80 \%$.

\begin{tabular}{cc} 
Patients Satisfaction & No. (\%) \\
Excellent & $\mathrm{n}=20(80 \%)$ \\
less pain and discomfort & $\mathrm{n}=3(12 \%)$ \\
persistent pain, Seroma and Parasthesia & $\mathrm{n}=2(8 \%)$ \\
(Necrosis) Requires Second operation & \\
\hline
\end{tabular}


In this study most of our patients defect sizes ranged from $(5$ to $10 \mathrm{~cm})$ with no recurrence rate in the early postoperative period (2 years).

The anterior rectus sheath is one of the major components maintaining the integrity of the abdominal wall and contour; consequently many authors published various flap advancement to restore the abdominal wall. All these aimed to preserve this structure completely and have been evaluated [8]. These flaps preserve the anterior rectus sheath and are considered to be a result of denervation .with high incidence of bleeding and seroma.

The principal idea of any repair should be to reconstruct the abdominal wall integrity with closure of the fascial defect, as we planned in this study after anatomical fascial defect repair we applied the mesh above the external oblique and on both sides, without the need for muscle transfer, our technique incorporates the use of a large mesh reinforced with bilateral external oblique muscle aponeurosis.

Ramirez et al. [6] described a component separation technique which allowed a midline advancement of the abdominal wall of up to $10 \mathrm{~cm}$ on each side, without the need for musculofascial flaps. Moreover, this technique provides an innervated and vascularized compound for dynamic support by dividing the abdominal wall components along a vascular plane.

Regarding to postoperative complications, hematomas, seroma and recurrence rates we had no recurrence in all our cases, like the results published by other authors, who reported similar recurrence rates, hematoma, seroma and skin necrosis [9]. No superior results were reported for the mesh group in regards to wound infection requiring reoperation.

The used mesh was introduced after shaping it like an outstretched hand fingers directed toward the midline and continued with the other site mesh in an interrupted suture and placed its origin on the most lateral aspect of the external oblique or anterior rectus sheath.

In this study regarding the patients satisfaction of abdominal contouring most of our patients were satisfied with an excellent satisfaction, 20/25 (80\%) the results agree with published article by Davison et al. at 2009 [9]. They stated that repairs with and without body contouring techniques as part of the reconstructive plan had equivalent recurrence rates $(7.7 \%$ vs. $0 \%)$ and complication rates (31.7\% vs. $53 \%)$. Mean patient satisfaction was 4.8 of 5 .

The idea of mesh placement in the midline was abandoned owing to the fact that, an abdominal wall defect that had been created due to the huge ventral hernia, and this made a collagen defect unlikely thus in our work after plication and anchoring the mesh at the linea alba.

The aim of mesh placement laterally was to augment the thinned lateral abdominal wall, to prevent any possible postoperative bulging of the internal oblique and transverse muscles. Agree the published study done by Daniel et al. 2008 [10] [11], also we did not use intra-peritoneal meshes for the same contouring reasons, to avoid extensive adhesion formation [11].

Complications associated with abdominoplasty occur primarily in patients who are smokers, and, diabetics. In addition to the general postoperative complications such as bleeding, wound infection, delayed wound healing, skin or fat necrosis, numbness to abdominal region or thighs can occur but no recurrence rate was gained in repairing the hernia defect associated with body contouring that agree the result of Davison et al. 2009 [9], they stated that reconstruction of complex and recurrent hernias can be successfully performed with good patients satisfaction and equivalent complication rate, and concomitant body contouring surgery does not impact recurrence or complication rates and may contribute to reconstructive success.

Our procedure is most appropriate for patients who are already at their ideal sustainable weight. It also eliminates the need of a second surgery and the risk of anesthesia.

\section{Conclusion}

Reconstruction of the abdominal wall and contouring of the body is possible and maintainable by using the technique of a polypropylene mesh prosthesis mesh placement and augmentation to reinforce both recti longitudinally and strip the mesh in a hand fingers like processes to reinforce the thinned lateral abdominal wall.

\section{References}

[1] Fowler, M.E. (1994) Body Contouring Surgery. Nursing Clinics of North America, 29, 753.

[2] Guerra, A.B., Metzinger, S.E., Bidros, R.S., Rizzuto, R.P., Gill, P.S., Nguyen, A.H., Dupin, C.L. and Allen, R.J. (2004) Bilateral Breast Reconstruction with the Deep Inferior Epigastric Perforator (DIEP) Flap: An Experience with 280 
Flaps. Annals of Plastic Surgery, 52, 246-252. http://dx.doi.org/10.1097/01.sap.0000110529.37143.96

[3] Özbek, S. and Özcan, M. (2005) Umbilicus Reconstruction with Modified "Unfolded Cylinder" Technique. British Journal of Plastic Surgery, 58, 500-503. http://dx.doi.org/10.1016/j.bjps.2004.11.013

[4] Avelar, J. (1989) Regional Distribution and Behaviour of the Subcutaneous Tissue Concerning Selection and Indication for Liposuction. Aesthetic Plastic Surgery, 13, 155-166. http://dx.doi.org/10.1007/BF01570212

[5] Rozen, W.M., Kapila, S., Enajat, M., Tan, M.J., Whitaker, I.S. and Acosta, R. (2012) Autologous Dermal Grafts for Rectus Sheath Reconstruction and Application in Closure of Ventral Myofascial Defects. Updates in Surgery, 64, 203210. http://dx.doi.org/10.1007/s13304-012-0167-y

[6] Ramirez, O.M., Ruas, E. and Dellon, A.L. (1990) “Components Separation” Method for Closure of Abdominal-Wall Defects: An Anatomic and Clinical Study. Plastic and Reconstructive Surgery, 86, 519-526. http://dx.doi.org/10.1097/00006534-199009000-00023

[7] Satterwhite, T.S., Miri, S., Chung, C., Spain, D.A., Lorenz, H.P. and Lee, G.K. (2012) Abdominal Wall Reconstruction with Dual Layer Cross-Linked Porcine Dermal Xenograft: The "Pork Sandwich" Herniorraphy. Journal of Plastic, Reconstructive \& Aesthetic Surgery, 65, 333-341. http://dx.doi.org/10.1016/j.bjps.2011.09.044

[8] Mathes, S.J., Steinwald, P.M., Foster, R.D., Hoffman, W.Y. and Anthony, J.P. (2000) Complex Abdominal Wall Reconstruction: A Comparison of Flap and Mesh Closure. Annals of Surgery, 232, 586-596.

[9] Davison, S.P., Parikh, P.M., Jacobson, J.M., Iorio, M.L. and Kalan, M. (2009) A “Buttressed Mesh” Technique for Fascial Closure in Complex Abdominal Wall Reconstruction. Annals of Plastic Surgery, 62, 284-289. http://dx.doi.org/10.1097/SAP.0b013e31817e9c6d

[10] Kaemmer, D.A., Conze, J., Otto, J. and Schumpelick, V. (2008) New Technical Approach for the Repair of an Abdominal Wall Defect after a Transverse Rectus Abdominis Myocutaneous Flap: A Case Report. Journal of Medical Case Reports, 2, 108. http://dx.doi.org/10.1186/1752-1947-2-108

[11] Rasim, Z.M., Alzahrani, M.A., Sigman, H.H., Meakins, J.L. and, Fried, G.M. (1997) Comparison of Adhesion Formation and Tensile Strength after Three Laparoscopic Herniorrhaphy Techniques. Surgical Laparoscopy Endoscopy \& Percutaneous Techniques, 7, 133-136. http://dx.doi.org/10.1097/00019509-199704000-00013 